\title{
Dysregulation of microRNAs as the risk factor of lymph node metastasis in papillary thyroid carcinoma: systematic review
}

\author{
Romena Laukiene', Valentinas Jakubkevicius², Laima Ambrozaityte', Loreta Cimbalistiene', \\ Algirdas Utkus ${ }^{1}$ \\ ${ }^{1}$ Department of Human and Medical Genetics, Institute of Biomedical Sciences, Faculty of Medicine, Vilnius University, Vilnius, \\ Lithuania \\ ${ }^{2}$ Faculty of Medicine, Vilnius University, Vilnius, Lithuania
}

\begin{abstract}
Papillary thyroid carcinoma (PTC) has an excellent prognosis with a relatively low mortality rate, but a small portion of PTC patients suffer from an aggressive form of the disease. In such cases early detection of lymph node metastasis (LNM) is as paramount as it is problematic. The routine use of central neck lymph node dissection is not recommended. New methods to detect LNM are needed. MicroRNAs are a potential biomarker for diagnosis and prognosis of PTC. In this review we summarise the current knowledge regarding dysregulated miRNAs and their association with LNM in PTS patients.

The PubMed and EBSCO databases were searched using terms for "microRNA", "thyroid carcinoma", and "prognosis" by using Boolean operators. Based on eligibility and exclusion criteria, articles were screened and reviewed in full, methodological data of included studies were extracted, and risk of bias analysis performed.

In total, 446 unique studies were extracted from the mentioned databases, and based on inclusion and exclusion criteria 27 studies were included in this review. Of them 17 analysed tissue microRNAs, 5 analysed circulating microRNAs, and 5 studies analysed both tissue and circulating samples. MiRNA-146B, miRNA-221, miRNA-222, miRNA-21, miRNA-204, miRNA-451, miRNA-199a-3p, and miRNA-30a-3p were dysregulated in at least 2 separate studies. A sizable portion of studies failed to show statistically significant differences in miRNA expression between LNM-positive and -negative patients. Different methodologies and disparities of patient populations could explain these discrepancies.

This research supports the statement that specific up- and downregulated miRNAs are associated with LNM in PTC patients. However, the prognostic value of these miRNAs is limited. Additional targeted cohort studies are required to elucidate the role of miRNAs in defining individualised treatment strategies for thyroid cancer patients. (Endokrynol Pol 2021; 72 (2): 145-152)
\end{abstract}

Key words: thyroid neoplasms; microRNAs; lymphatic metastasis

\section{Introduction}

Thyroid cancer is the most frequently occurring endocrine malignancy, with an increasing rate of incidence over the last 3 decades [1]. It accounts for $1.7 \%$ of all malignant tumours worldwide [2]. Papillary thyroid carcinoma (PTC) accounts for up to $85 \%$ of all thyroid cancers [3]. Generally, PTC has an excellent prognosis with a relatively low mortality rate, but a small portion of PTC patients suffer from an aggressive form of the disease, with tumour invasion and metastasis [4]. The detection of neck lymph node metastasis (LNM) - especially subclinical — of thyroid cancer using imaging methods (ultrasound, computer tomography, magnetic resonance imaging) is often problematic [5]. In up to $60 \%$ of imaging lymph node-negative patients, cervical
LNM are found on histology [6]. This could result in incomplete clinical treatment. The American Thyroid Association Management guidelines recommend the use of central neck dissection for patients with clinically involved central nodes, but the use of prophylactic central node dissection ( $\mathrm{pCND}$ ) in patients without clinically detected central neck lymph nodes remains controversial [7]. Prophylactic central node dissection significantly reduces locoregional recurrence in PTC patients with clinically uninvolved central neck lymph nodes, but $\mathrm{pCND}$ is often associated with postoperative complications. Patients with pCND have significantly higher chances of transient recurrent laryngeal nerve injury and transient or permanent hypocalcaemia [8]. Therefore, accurate identification of LNM is of crucial importance in optimising individualised PTC treatment. 
Table 1. Detailed search retrieval from PubMed and EBSCO databases, including results for terms and their combinations

\begin{tabular}{|c|c|c|c|c|}
\hline No. & Search details in PubMed & Search details in EBSCO & PubMed & EBSCO \\
\hline 1 & $\begin{array}{l}\text { "thyroid cancer" [Title/Abstract] OR "thyroid carcinoma" } \\
\text { [Title/Abstract] OR "thyroid nodule" [Title/Abstract] } \\
\text { OR thyroid neoplasms [MeSH Terms] }\end{array}$ & $\begin{array}{l}\text { (TI thyroid cancer OR AB thyroid cancer) } \\
\text { OR (TI thyroid carcinoma } \\
\text { OR AB thyroid carcinoma) } \\
\text { OR (TI thyroid nodule } \\
\text { OR AB thyroid nodule) }\end{array}$ & 63,540 & 76,736 \\
\hline 2 & $\begin{array}{l}\text { "micro RNA" [Title/Abstract] OR “miRNA” } \\
\text { [Title/Abstract] OR „miR” [Title/Abstract] } \\
\text { OR micrornas [MeSH Terms] }\end{array}$ & $\begin{array}{l}\text { (TI micro RNA OR AB micro RNA) } \\
\text { OR (TI miRNA OR AB miRNA) } \\
\text { OR (TI miR OR AB miR) }\end{array}$ & 114,487 & 134,624 \\
\hline 3 & $\begin{array}{l}\text { "prognosis" [Title/Abstract] OR "prognos* } \\
\text { "[Title/Abstract] OR “lymph node" [Title/Abstract] } \\
\text { OR "metasta*" [Title/Abstract] } \\
\text { OR prognosis [MeSH Terms] }\end{array}$ & $\begin{array}{l}\text { (TI prognos* OR AB prognos*) } \\
\text { OR (TI lymph node OR AB lymph node) } \\
\text { OR (TI metasta* OR AB metasta*) }\end{array}$ & $2,330,377$ & $1,670,450$ \\
\hline 4 & 1 AND 2 & 1 AND 2 & 918 & 890 \\
\hline 5 & 3 AND 4 & 3 AND 4 & 410 & 359 \\
\hline
\end{tabular}

Many researchers are looking for new testing methods to identify LNM before surgery. Advanced new molecular genetic biomarkers could identify patients with aggressive PTC for more aggressive treatment options.

MicroRNAs (miRNAs) are a class of non-coding RNAs approximately 19-24 nucleotides in length that can function as oncogenes or tumour suppressors by inhibiting the translation of tumour suppressor genes or by blocking the translation of oncogenes [9]. MicroRNAs modify gene expression by binding to specific targets in the $3^{\prime}$ untranslated region, and they are implicated in tumourigenesis of a variety of tissues [10]. Regulation of classical oncogenes and tumour suppressor genes by miRNAs was easily identified as a hallmark of cancer research, transforming this class of small RNAs into potential targets for cancer diagnosis, prognosis, and therapy. With the availability of high-throughput, next-generation sequencing, miRNAs can be detected more accurately from controls compared with other miRNA microarrays, northern blots, and TaqMan microRNA Assays Human panel [11]. There has been a lot of interest in the feasibility of miRNAs as biomarkers for the diagnosis of thyroid cancer in recent years. Several miRNAs possibly associated with LNM were identified in patients with PTC [12].

In this review, we focus on miRNA expression in thyroid cancer to evaluate miRNA signatures associated with LNM. The objective of this systematic review is to summarise the current knowledge regarding dysregulated miRNAs and their association with LNM in patients with PTC.

\section{Systematic literature analysis}

Systematic literature analysis was performed following the Cochrane Handbook for Interventional Systematic
Reviews. The Study was written in accordance with the guidelines proposed by the preferred reporting items for systematic review and meta-analyses (PRISMA) [13]. Search was performed by querying the PubMed and EBSCO databases with terms for "micro RNA", "thyroid carcinoma", and "prognosis" by using Boolean operators, for articles published prior to 2020-08-13 (Tab. 1). Two reviewers (R.L., V.J.) working independently using standardised forms screened titles and abstracts to identify potentially relevant studies. Articles of interest were further evaluated in their entirety (Fig. 1).

Studies were considered to be eligible if they: focused on patients with any type of papillary thyroid carcinoma or had information about clinicopathological patient characteristics. The effect of the dysregulated miRNA on cervical lymph node metastases was assessed. Exclusion criteria consisted of the following: non-PTC thyroid cancer studies, non-English articles, reviews, letters, comments, and studies using miRNAs only for differentiation between benign and malignant thyroid lesions or studies with a primary focus on biomolecular mechanisms of miRNAs.

It is of note, reviewers elected not to exclude studies if they satisfied inclusion criteria but failed to find statistically significant differences in microRNA expression among LNM positive and negative groups.

Risk of bias was assessed using a quality assessment tool for diagnostic accuracy studies (QUADAS-2). QUADAS-2 focuses on the quality of primary diagnostic accuracy studies, but not on the patient cohorts, control groups, or blinding procedures. It consists of 4 key domains covering patient selection, index test (in our case miRNA), reference standard, and flow of patients through the study as well as the timing of the index test and reference standard ("flow and timing"). These domains are assessed in terms of risk of bias 


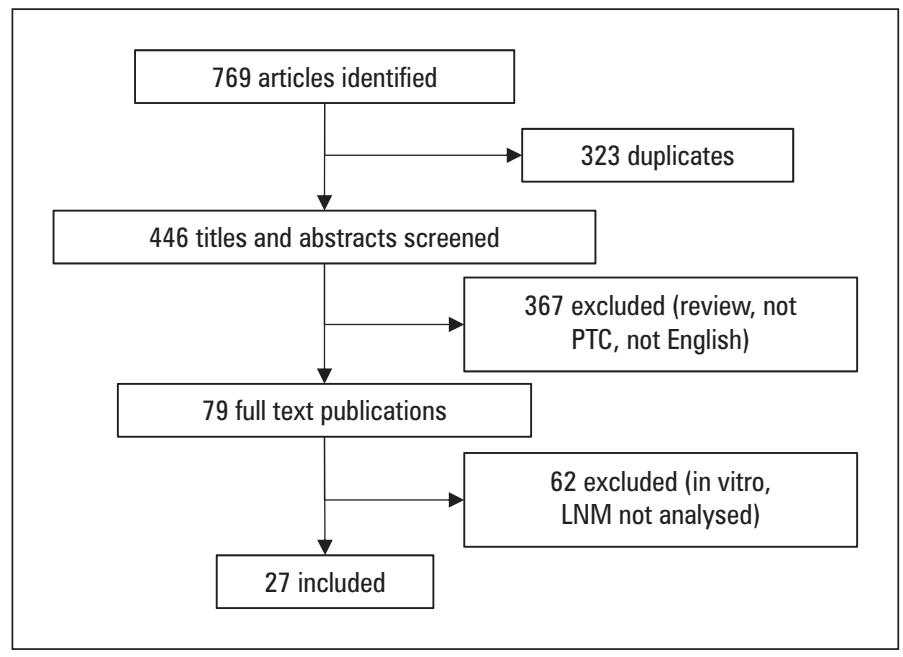

Figure 1. Flowchart of study selection. PTC — papillary thyroid carcinoma; LNM — lymph node metastasis

and the first 3 are also assessed in terms of concerns regarding applicability. To help reach a judgement on the risk of bias, signalling questions were included [14]. QUADAS-2 review questions and specific guidance for this review were discussed among the reviewers, and then evaluation was performed by 2 reviewers working separately.

Methodological and outcome data were extracted from the selected studies. Discrepancies between 2 reviewers in study selection (title and abstract screening, full article evaluation), data extraction, and risk of bias evaluation processes were discussed and a joint resolution was made.

\section{Analysis results}

The PubMed search yielded 410 possible studies and EMBSCO yielded 359. In total 446 unique studies were included in the further analysis after removing duplicates. Based on the inclusion and exclusion criteria, 367 studies were excluded after title and abstract screening (most were reviews and studies not addressing PTC or lacking clinicopathological data), and when the decision was not clear, the studies were analysed in full. Of the 79 full text publications, 27 studies were included in this review (Fig. 1). The 2 reviewers were in agreement regarding study eligibility. The earliest study was published in May 2010 [15] and the latest in June 2020 [16]. The included studies were from 7 countries: China $(n=20)$, Serbia $(n=2)$, Lithuania $(n=1)$, South Korea $(\mathrm{n}=1)$, Taiwan $(\mathrm{n}=1)$, Turkey $(\mathrm{n}=1)$, and the USA $(n=1)$.

\section{Study design}

A methodological summary of the included studies is summarised in Supplementary File - Table S1.
From 27 studies, 17 analysed tissue microRNAs, 5 analysed circulating microRNAs, and 5 studies analysed both tissue and circulating samples. Among studies analysing tissue, 11 evaluated freshly frozen (snap-frozen) samples, 9 analysed miRNAs from formalin-fixed paraffin-embedded tissue (FFPE), one analysed tissue from fine needle aspiration (FNA), and in 1 case the method of specimen preparation/extraction was not specified. In studies analysing circulating microRNAs, 3 analysed plasmas, 4 - serum, 1 - blood (otherwise not specified), 1 - plasma exosomes, and 1 - both plasma and serum exosomes (Fig. 2).

For initial genome screening, 4 studies [15, 20-22] performed microarray, and 1 study [17] used Solexa sequencing, followed by qRT-PCR to validate results. However, of all the rest of the studies 21 performed qRT-PCR [16, 19, 23-41] and 1 study performed northern blotting [18] to quantify miRNA expression for a priori selected miRNAs from the literature.

The studies employed a variety of statistical methods. Twenty-one studies compared continuous miRNA expression between different LNM groups. When parametric test assumptions were met, Student's t-test and ANOVA were used, and when such assumptions were not met, Kruskal-Wallis $\mathrm{H}$ and Mann-Whitney $\mathrm{U}$ tests were used. Analysing associations between miRNA and LNM, one study [17] used Pearson's correlation coefficient to correlate miRNAs from blood and tissue, and 2 studies $[26,33]$ used multivariate logistic regression to assess independent predictors for LNM. Six studies divided miRNA expression into low/high groups based on mean or median values, and then compared those groups as categorical variables using the chi-square test to assess differences between miRNA expression and LNM status [16, 28-31, 37]. Four studies [32, 38, 39, 41] performed ROC analysis to evaluate the diagnostic utility of miRNA 


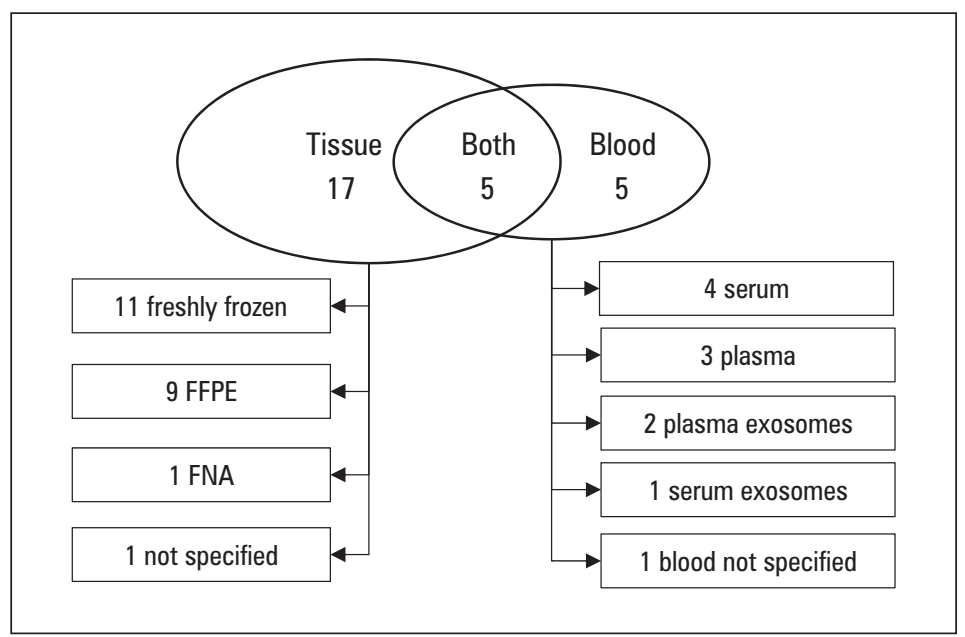

Figure 2. Studies by analysed specimens. FFPE — formalin-fixed paraffin-embedded tissue; FNA — fine needle aspiration

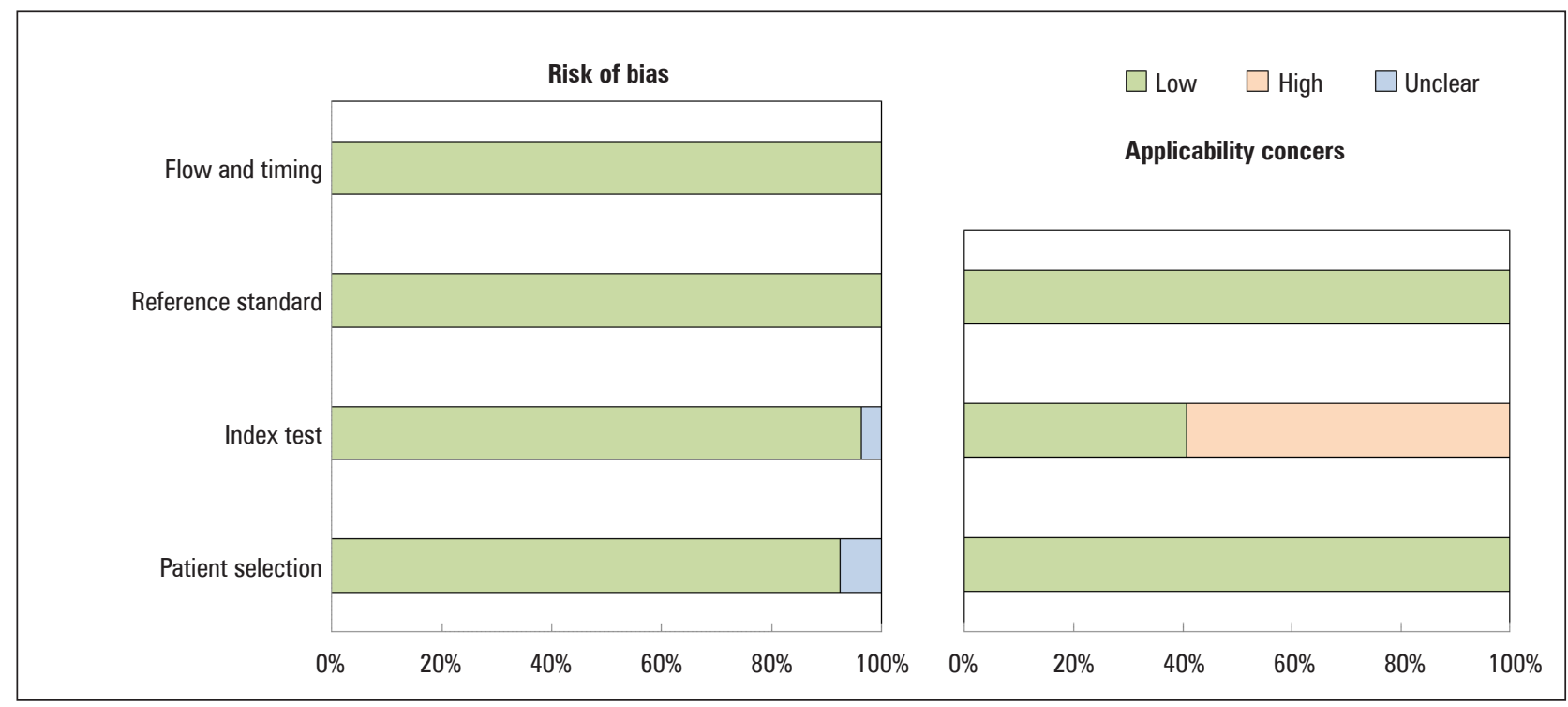

Figure 3. Summary of risk of bias analysis by quality assessment tool for diagnostic accuracy studies (QUADAS-2)

in predicting LNM. One study [38] used univariate and multivariate Cox proportional hazard model to analyse miRNA as independent risk factors for LNM.

A summary of the risk of bias analysis can be found in Supplementary File - Table S2, and Figure 3.

Most of the studies included in the analysis were at low risk of bias in all evaluated domains. However, in some studies the patient population and methodological descriptions were not fully clear. Several previous studies showed discrepancies between miRNA profiles in surgically resected tissues and FNA material, which led other authors to conclude that the results from surgically resected material cannot be extrapolated into preoperative use without further validation [42]. Therefore, this review attempted to find miRNA able to inform the extent of surgery, hence changes in
miRNA from post-surgical specimens may not necessarily translate to changes measurable preoperatively (e.g. serum, FNA specimens). Additionally, all but one of the included studies performed clinically indicated or not specified cervical lymph node dissection, as opposed to prophylactic cervical lymph node dissection, and this could lead to a selection bias, favouring more advanced disease and missing early stages of cervical LNM. This could also explain why in a sizable portion of included studies there were more PTC patients with LNM than without LNM.

Of note, only one [26] of the included studies specified PTC subtype in a LNM vs. no LNM context, providing separate analysis for all variants combined and classical variant in particular excluding Tall cell and follicular PTCs. 
Table 2. Dysregulated miRNA mentioned in at least 2 separate studies

\begin{tabular}{lccc}
\hline MiR & Studies with $\uparrow$ MiR & Studies with $\downarrow$ MiR & No statistically significant difference \\
\hline $146 \mathrm{~b}$ & {$[24, \mathbf{2 6}, \mathbf{3 4}, \mathbf{3 8}, 40]$} & {$[15, \mathbf{1 9}, \mathbf{2 0}, \mathbf{2 2}, \mathbf{2 3}, \mathbf{2 5}]$} \\
\hline 221 & {$[18,19,24, \mathbf{2 6}, \mathbf{3 8}, 40]$} & {$[15, \mathbf{1 8}, \mathbf{2 0}, \mathbf{2 3}, \mathbf{2 6}]$} \\
\hline 222 & {$[\mathbf{1 7}, \mathbf{1 9}, \mathbf{2 4}, \mathbf{2 6}, \mathbf{3 6}, \mathbf{3 8}, 40]$} & {$[15, \mathbf{2 0}, \mathbf{2 3}, 36]$} \\
\hline 21 & {$[\mathbf{3 8}, 40]$} & {$[19.26]$} \\
\hline 204 & {$[\mathbf{3 8}]$} & {$[26, \mathbf{3 9}]$} \\
\hline 451 & {$[21]$} & {$[\mathbf{3 2}]$} & \\
\hline $199 \mathrm{a}-3 p$ & & {$[31]$} & {$[\mathbf{3 8}]$} \\
\hline $30 \mathrm{a}-3 p$ & & {$[22,35, \mathbf{3 8}]$} \\
\hline
\end{tabular}

bold - specimens could be obtained preoperatively

\section{MicroRNA expression and LNM}

Dysregulated miRNAs associated with LNM in the included studies are listed in Supplementary Table S3 (with pre-operative specimens in bold). When the same miRNA was mentioned in at least 2 separate studies, they were listed in Table 2 (specimens obtained preoperatively are shown in bold).

miRNA-146B, miRNA-221, miRNA-222, miRNA-21, miRNA-204, miRNA-451, miRNA-199a-3p, and miRNA-30a-3p were mentioned in at least 2 separate studies. Similar at least two different studies excluding tissue specimen miRNA-221, miRNA-222, miRNA-146b, and miRNA-204 were found as well. Some studies analyzed not only differences between LNM-positive and -negative groups, but also tried to find differences in miRNA expression between groups based on LNM location in the neck lateral LNM vs. central LNM $[21,23,36]$.

Most of the studies agreed on the presents of miRNA dysregulation; however, in some studies statistically significant differences were not identified (Tab. 2).

In one study upregulated miRNA-451 was detected in patients with LNM [21] and downregulated by another one [32].

The same trend remains even in studies where specimens were available preoperatively. This could be explained by different methodologies, groups sizes, and disparities in patient populations, because all but one of the studies lymphadenectomies were performed only by clinical suspicion or the indication was not specified.

\section{miRNA biomarkers predicting LNM in PTC}

Differentiated thyroid cells express a pool of miRNAs, alterations in the expression of which could induce neoplastic changes. A downregulation of miRNAs that ensure normal function of cells, or alternatively an upregulation of miRNAs that promote oncogenic effects, can lead to increased expression of oncogenes. That can result in the induction of the malignant effects of cell proliferation, differentiation, and apoptosis, leading to tumour growth and progression [43]. In this systematic review, we focused on exploring the utility of miRNA biomarkers predicting LNM in PTC.

\section{MicroRNA-146}

MicroRNA-146 is one of the most investigated miRNAs in thyroid cancer and has been shown to be reliably upregulated in PTC $[24,26,34,38,40]$.

Functional analyses of miRNA-146 revealed its involvement in various cellular functions including proliferation, migration, and invasion [44, 45]. Chou et al. demonstrated that miRNA-146b expression was an independent risk factor for poor prognosis in PTC [46].

Chen et al. indicated that microRNAs can be identified not only in tissue samples but also in serum and plasma in a remarkably stable form. That makes miRNA expression detection possible in blood samples and serum, serving as potential biomarkers to detect various cancers. [47]. The clinical utility of using miRNAs as a biomarker in serum could be a diagnostic tool for identifying patients with LNM in PTC for more aggressive treatment options.

Eleven articles in our review described the association between miRNA-146 overexpression and LNM in PTC. Eight of these studies analysed tissue miRNA (4 FFPE, 3 FF, and 1 FNA) [15, 19, 20, 22, 24, 26, 34, 40], 2 - circulating samples (plasma exosome) [23, 38] and $1-$ both tissue and circulating samples (FF and blood) [25].

Several independent groups of investigators examining microRNA in histological samples found the expression level of miRNA146b to be significantly elevated in patients with lymph node metastases [24, 26, 40]. However, no significant correlation was observed in other studies $[15,19,20,22]$.

Sun et al. found positive associations between levels of miRNA-146a expression in PTC tissues and 
positive cervical lymph node metastasis. Nevertheless, the same study did not note significant differences in expression of miRNA-146b in the peripheral blood or in tissue between patients with PTC and LNM and patients with PTC but without LNM, which indicates that miRNA-146a and miRNA-146b expression in peripheral blood are not useful markers for LNM in PTC [25].

Yang et al. examined miRNA-146b expression in FNA, and malignancy was confirmed by histology, as a gold standard for accuracy. That study showed that levels of miR146b were significantly higher in PTCs with lymph node metastasis [34].

Jiang et al. explored the selected exosomal miRNAs as potential biomarkers predicting LNM in PTCs. Exosomal miRNA-146b-5p and miRNA-222-3p ROC analysis showed AUCs of 0.811 and 0.834 , respectively. Combining exosomal miRNA-146b-5p and miRNA-222-3p significantly improved the diagnostic value and increased the AUC to 0.895 , with a sensitivity and specificity of $85.1 \%$ and $80.0 \%$, respectively [38].

The only study in our review by Han et al. examined miRNA expression in the patients undergoing prophylactic lymph node dissection. The specimens they used were FFPE. On FFPE specimens miRNA-146b-3p, miRNA-146b-5p, and miRNA-222 were identified as potential markers of LNM [26].

\section{miRNA-221/222}

Many PTC studies in our review identified expression of miRNA-221/222 as the most consistently upregulated miRNAs, with significant association with clinicopathological features. PTC with LNM showed higher tissue miRNA-221/222 expression in comparison to PTC without LNM [18, 19, 24, 26, 36, 40]. Jiang et al. revealed that a high serum miRNA-222 and miRNA-221 level has significant correlation with the presence of LNM [38]. Enhanced expression of serum miRNA-222 was also found in patients with cervical lymph node metastasis in a study by Yu et al. [17]. In an another study, Lee et al. [23] recruited PTC patients with and without LNM. Mean fold changes in plasma-derived miRNA-221 $(-4.86 \pm 0.051)$, and miRNA-222 $(-5.36 \pm 0.91)$ in patients with LNM group were slightly higher than that of miR-221 (-5.06 \pm 0.074$)$, and miR-222 (-5.45 \pm 0.079$)$ in patients without LNM. However, without statistical significance.

\section{miRNA-451}

Another important member of the microRNA family is miRNA-451, which has been shown to be downregulated in various human tumours [48]. It is dysregulated in multiple cancers and take part in various human physiological and pathological processes and cancer-related biological processes such as apoptosis, angiogenesis, proliferation, and metastasis [49-51]. It often acts as a tumour suppressor gene in various cancers. However, little is known about its role in PTC.

In the reviewed articles we found that the data about miRNA-451 association with LNM in PTC are controversial. The results of a study by Zhang et al. [32] indicated a strong correlation with lower miR-451 levels in PTC patients with LNM, suggesting that the tissue miRNA-451 level is also a "good" biomarker (AUC $=0.792)$ for lymph node metastasis. Expression of serum miR-451 in positive LNM patients' samples was only modestly $(77 \%)$ lower than in LNM-negative patients. ROC analysis of serum miRNA-451 as a biomarker for evaluation of lymph node status was performed. A value of 0.690 in AUC would indicate that it is a possible "modest" biomarker for lymph node metastasis.

Conversely, the findings of Wang et al. [21] revealed that the expression of miRNA-451 tissue was significantly upregulated in PTC with LNM, compared to PTC cases without LNM. These findings suggest an oncogenic role for miRNA-451 in PTC. Further studies in larger cohorts are needed to explain the discrepant results on the role of miRNA-451 in thyroid cancer.

\section{MicroRNA-199a-3p}

MicroRNA-199a-3p is downregulated in a number of different cancers, such as ovarian carcinoma, colorectal cancers, and hepatocellular carcinoma [52-54]. The results of a study by Liu et al. [31] showed positive associations between levels of miRNA-199a-3p expression in PTC tissues and positive cervical lymph node metastasis.

The same study revealed that low miR-199a-3p expression was associated with LNM $(p=0.036)$ and recurrence of LNM ( $p=0.03)$. These results suggest that the detection of miRNA-199a-3p in the PTC could reflect metastasis and predict prognosis.

Jiang et al. [38] recruited 136 patients with PTC. The expression level of serum miRNA-199a-3p was examined in patients with and without LNM. However, the difference between groups was not significant.

One of the factors limiting the comparison of miRNA changes between different publications is that most studies use their own rather subjective miRNA expression values, and no absolute levels of target miRNAs are set, which complicates the external validity usage of miRNA as a clinical tool.

In addition, as was noted in another review [55], analysis of miRNA expression and underlying mechanisms cannot be related to a single miRNA analysis. Due to non-canonical and partially complementary binding properties of miRNA, nearly half of the miRNA targets contain binding sites for at least 2 miRNAs. Therefore, 
miRNAs binding to the same target can synergise and/or antagonise the expression of target genes, making studies on single miRNAs questionable.

As discussed by Han et al. [26], statistical significance does not necessarily predict the actual clinical applicability of molecular markers. The authors found that 3 miRNAs were able to predict LNM in a multivariate model. However, there was significant overlap in their expression in LNM-positive and LNM-negative groups. Only in a minority of cases were miRNA expression levels high enough to enable segregation of LNM-positive cases, providing poor sensitivity at acceptable levels of specificity.

\section{Conclusions}

This research supports the statement that specific upand downregulated miRNAs are associated with LNM in PTC patients. However, the prognostic value of these miRNAs is limited in individual cases because the distribution of miRNA expression overlaps between patients with LNM and without LNM. Analysis of miRNA expression levels and detection of circulating or FNA miRNAs can be used for the pre-operative diagnosis of thyroid cancer cervical lymph node metastasis. To translate these data into clinical application, large cohort studies are required to examine the prognostic and diagnostic value of miRNAs panels. Improved standardisation of methods used to assay miRNAs will allow more extensive use of this approach in defining individualised treatment strategies for thyroid cancer patients.

\section{References}

1. Pellegriti G, Frasca F, Regalbuto C, et al. Worldwide increasing incidence of thyroid cancer: update on epidemiology and risk factors. J Cance Epidemiol. 2013; 2013: 965212, doi: 10.1155/2013/965212, indexed in Pubmed: 23737785.

2. Ferlay J, Shin HR, Bray F, et al. Estimates of worldwide burden of cancer in 2008: GLOBOCAN 2008. Int J Cancer. 2010; 127(12): 2893-2917, doi: 10.1002/ijc.25516, indexed in Pubmed: 21351269

3. Davies L, Welch HG. Increasing incidence of thyroid cancer in the United States, 1973-2002. JAMA, 2006; 295(18): 2164-2167, doi: 10.1001/jama.295.18.2164, indexed in Pubmed: 16684987.

4. Cho SW, Choi HS, Yeom GJ, et al. Long-term prognosis of differentiated thyroid cancer with lung metastasis in Korea and its prognostic factors. Thyroid. 2014; 24(2): 277-286, doi: 10.1089/thy.2012.0654, indexed in Pubmed: 23758653.

5. Tao Y, Wang C, Li L, et al. Clinicopathological features for predicting central and lateral lymph node metastasis in papillary thyroid microcarcinoma: Analysis of 66 cases that underwent central and lateral lymph node dissection. Mol Clin Oncol. 2017; 6(1): 49-55, doi: 10.3892/mco.2016.1085, indexed in Pubmed: 28123728.

6. Wada N, Duh QY, Sugino K, et al. Lymph Node Metastasis From 259 Papillary Thyroid Microcarcinomas. Ann Surg. 2003; 237(3): 399-407, doi: 10.1097/01.sla.0000055273.58908.19, indexed in Pubmed: 12616125.

7. Haugen BR. 2015 American Thyroid Association Management Guidelines for Adult Patients with Thyroid Nodules and Differentiated Thyroid Cancer: What is new and what has changed? Cancer. 2017; 123(3): 372-381, doi: 10.1002/cncr.30360, indexed in Pubmed: 27741354

8. Chen L, Wu YH, Lee CH, et al. Prophylactic Central Neck Dissection for Papillary Thyroid Carcinoma with Clinically Uninvolved Centra Neck Lymph Nodes: A Systematic Review and Meta-analysis. World
J Surg. 2018; 42(9): 2846-2857, doi: 10.1007/s00268-018-4547-4, indexed in Pubmed: 29488066.

9. Tan W, Liu B, Qu S, et al. MicroRNAs and cancer: Key paradigms in molecular therapy. Oncol Lett. 2018; 15(3): 2735-2742, doi: 10.3892/ol.2017.7638, indexed in Pubmed: 29434998.

10. Bartel DP. MicroRNAs: genomics, biogenesis, mechanism, and function Cell. 2004; 116(2): 281-297, doi: 10.1016/s0092-8674(04)00045-5, indexed in Pubmed: 14744438 .

11. Santiago K, Chen Wongworawat $Y$, Khan S. Differential MicroRNA-Signatures in Thyroid Cancer Subtypes. J Oncol. 2020; 2020: 2052396, doi: 10.1155/2020/2052396, indexed in Pubmed: 32565797.

12. Ab Mutalib NS, Othman SN, Mohamad Yusof A, et al. Integrated microRNA, gene expression and transcription factors signature in papillary thyroid cancer with lymph node metastasis. PeerJ. 2016; 4: e2119, doi: 10.7717/peerj.2119, indexed in Pubmed: 27350898

13. Moher D, Liberati A, Tetzlaff J, et al. PRISMA Group, PRISMA Group, PRISMA Group, PRISMA Group, PRISMA Group, PRISMA Group, PRISMA Group. Preferred reporting items for systematic reviews and meta-analyses: the PRISMA Statement. Open Med. 2009; 3(3): e123-e130, indexed in Pubmed: 21603045.

14. Whiting PF, Rutjes AWS, Westwood ME, et al. QUADAS-2 Group. QUADAS-2: a revised tool for the quality assessment of diagnostic accuracy studies. Ann Intern Med. 2011; 155(8): 529-536, doi: 10.7326/0003-4819155-8-201110180-00009, indexed in Pubmed: 22007046.

15. Chou CK, Chen RF, Chou FF, et al. miR-146b is highly expressed in adult papillary thyroid carcinomas with high risk features including extrathyroidal invasion and the BRAF(V600E) mutation. Thyroid. 2010; 20(5): 489-494, doi: 10.1089/thy.2009.0027, indexed in Pubmed: 20406109.

16. Shi C, Cao J, Shi T, et al. BRAF mutation, BRAF-activated long non-coding RNA and miR-9 expression in papillary thyroid carcinoma, and their association with clinicopathological features. World I Surg Oncol. 2020 18(1): 145, doi: 10.1186/s12957-020-01923-7, indexed in Pubmed: 32593310.

17. Yu S, Liu Y, Wang J, et al. Circulating microRNA profiles as potential biomarkers for diagnosis of papillary thyroid carcinoma. J Clin Endocrinol Metab. 2012; 97(6): 2084-2092, doi: 10.1210/jc.2011-3059, indexed in Pubmed: 22472564

18. Zhou YL, Liu C, Dai Xx, et al. Overexpression of miR-221 is associated with aggressive clinicopathologic characteristics and the BRAF mutation in papillary thyroid carcinomas. Med Oncol. 2012; 29(5): 3360-3366, doi: 10.1007/s12032-012-0315-8, indexed in Pubmed: 22855362.

19. Sun $Y$, Yu S, Liu Y, et al. Expression of miRNAs in Papillary Thyroid Carcinomas Is Associated with BRAF Mutation and Clinicopathological Features in Chinese Patients. Int J Endocrinol. 2013; 2013: 128735, doi: 10.1155/2013/128735, indexed in Pubmed: 23690767.

20. Wang Z, Zhang H, He L, et al. Association between the expression of four upregulated miRNAs and extrathyroidal invasion in papillary thyroid carcinoma. Onco Targets Ther. 2013; 6: 281-287, doi: 10.2147/OTT.S43014, indexed in Pubmed: 23569392.

21. Wang Z, Zhang H, Zhang P, et al. Upregulation of miR-2861 and miR-451 expression in papillary thyroid carcinoma with lymph node metastasis. Med Oncol. 2013; 30(2): 577, doi: 10.1007/s12032-013-0577-9, indexed in Pubmed: 23609190.

22. Peng Y, Li C, Luo DC, et al. Expression profile and clinical significance of microRNAs in papillary thyroid carcinoma. Molecules. 2014 19(8): 11586-11599, doi: 10.3390/molecules190811586, indexed in Pubmed: 25100252

23. Lee YSe, Lim YS, Lee JC, et al. Differential expression levels of plasma-derived miR-146b and miR-155 in papillary thyroid cancer. Oral Oncol. 2015; 51(1): 77-83, doi: 10.1016/j.oraloncology.2014.10.006, indexed in Pubmed: 25456009

24. Acibucu F, Dökmetaş HS, Tutar Y, et al. Correlations between the expression levels of micro-RNA146b, 221, 222 and p27Kip1 protein mRNA and the clinicopathologic parameters in papillary thyroid cancers. Exp Clin Endocrinol Diabetes. 2014; 122(3): 137-143, doi: 10.1055/s-0034-1367025, indexed in Pubmed: 24643689.

25. Sun M, Fang S, Li W, et al. Associations of miR-146a and miR-146b expression and clinical characteristics in papillary thyroid carcinoma. Cancer Biomark. 2015; 15(1): 33-40, doi: 10.3233/CBM-140431, indexed in Pubmed: 25524940.

26. Han PA, Kim Hs, Cho S, et al. Association of BRAF V600E Mutation and MicroRNA Expression with Central Lymph Node Metastases in Papillary Thyroid Cancer: A Prospective Study from Four Endocrine Surgery Centers. Thyroid. 2016; 26(4): 532-542, doi: 10.1089/thy.2015.0378, indexed in Pubmed: 26950846

27. $\mathrm{Hu} \mathrm{J}, \mathrm{Li} \mathrm{C}$, Liu C, et al. Expressions of miRNAs in papillary thyroid carcinoma and their associations with the clinical characteristics of PTC. Cancer Biomark. 2017; 18(1): 87-94, doi: 10.3233/CBM-161723, indexed in Pubmed: 28085013.

28. Liu Na, Zhou Qi, Qi YH, et al. Effects of long non-coding RNA H19 and microRNA let7a expression on thyroid cancer prognosis. Exp Mol Pathol. 2017; 103(1): 71-77, doi: 10.1016/j.yexmp.2017.06.004, indexed in Pubmed: 28655518. 
29. Huang T, Yi D, Xu L, et al. Downregulation of miR-381 is associated with poor prognosis in papillary thyroid carcinoma. Int J Clin Exp Pathol. 2017; 10(12): 11610-11616, indexed in Pubmed: 31966518.

30. Ren G, Li H, He X, et al. Downregulation of serum miR-26a predicts poor clinical outcome of papillary thyroid carcinoma. Int J Clin Exp Pathol. 2017; 10(8): 9042-9047, indexed in Pubmed: 31966775.

31. Liu C, Xing M, Wang L, et al. miR-199a-3p downregulation in thyroid tissues is associated with invasion and metastasis of papillary thyroid carcinoma. Br J Biomed Sci. 2017; 74(2): 90-94, doi: 10.1080/09674845.20 16.1264705, indexed in Pubmed: 28367740.

32. Zhang M, Wu W, Gao M, et al. MicroRNA-451 as a prognostic marker for diagnosis and lymph node metastasis of papillary thyroid carcinoma. Cancer Biomark. 2017; 19(4): 437-445, doi: 10.3233/CBM-170059, indexed in Pubmed: 28582849.

33. Todorović L, Stanojević B, Mandušić V, et al. Expression of VHL tumor suppressor mRNA and miR-92a in papillary thyroid carcinoma and their correlation with clinical and pathological parameters. Med Oncol. 2018; 35(2): 17, doi: 10.1007/s12032-017-1066-3, indexed in Pubmed: 29340905.

34. Yang F, Zhang H, Leng X, et al. miR-146b measurement in FNA to distinguish papillary thyroid cancer from benign thyroid masses. $\mathrm{Br}$ J Biomed Sci. 2018; 75(1): 43-45, doi: 10.1080/09674845.2017.1347336, indexed in Pubmed: 28937915.

35. Todorović L, Mandušić V, Tadić BV, et al. Altered expression of microRNA-30a-3p in papillary thyroid cancer and its association with clinicopathological characteristics. Arch Biol Sci. 2020; 72: 31-36, doi: 10.2298/ABS191004063T.

36. Xiang D, Tian B, Yang T, et al. miR-222 expression is correlated with the ATA risk stratifications in papillary thyroid carcinomas. Medicine (Baltimore). 2019; 98(25): e16050, doi: 10.1097/MD.0000000000016050, indexed in Pubmed: 31232941.

37. Yao XG, Tan Q, Liu PP, et al. Tissue microRNA-182 expression level and its potential prognostic value for papillary thyroid carcinoma. Int J Clin Exp Pathol. 2019; 12(8): 3128-3133, indexed in Pubmed: 31934155.

38. Jiang Ke, Li G, Chen W, et al. Plasma Exosomal miR-146b-5p and miR-222-3p are Potential Biomarkers for Lymph Node Metastasis in Papillary Thyroid Carcinomas. Onco Targets Ther. 2020; 13: 1311-1319, doi: 10.2147/OTT.S231361, indexed in Pubmed: 32103998.

39. Dai D, Tan $Y$, Guo L, et al. Identification of exosomal miRNA biomarkers for diagnosis of papillary thyroid cancer by small RNA sequencing. Eur J Endocrinol. 2020; 182(1): 111-121, doi: 10.1530/EJE-19-0524, indexed in Pubmed: 31721725.

40. Pamedytyte D, Simanaviciene V, Dauksiene D, et al. Association of MicroRNA Expression and BRAF Mutation with Recurrence of Thyroid Cancer. Biomolecules. 2020; 10(4), doi: 10.3390/biom10040625, indexed in Pubmed: 32316638.

41. Liu Y, Li L, Liu Z, et al. Plasma miR-323 as a Biomarker for Screening Papillary Thyroid Cancer From Healthy Controls. Front Med (Lausanne). 2020; 7: 122, doi: 10.3389/fmed.2020.00122, indexed in Pubmed: 32478079.

42. Ludvíková M, Kalfeřt D, Kholová I. Pathobiology of MicroRNAs and Their Emerging Role in Thyroid Fine-Needle Aspiration. Acta Cytol. 2015; 59(6): 435-444, doi: 10.1159/000442145, indexed in Pubmed: 26745212.
43. Fuziwara CS, Kimura ET. MicroRNAs in thyroid development, function and tumorigenesis. Mol Cell Endocrinol. 2017; 456: 44-50, doi: 10.1016/j. mce.2016.12.017, indexed in Pubmed: 28011236.

44. Qiu Z, Li H, Wang J, et al. miR-146a and miR-146b in the diagnosis and prognosis of papillary thyroid carcinoma. Oncol Rep. 2017; 38(5): 2735-2740, doi: 10.3892/or.2017.5994, indexed in Pubmed: 29048684.

45. Lima CR, Geraldo MV, Fuziwara CS, et al. MiRNA-146b-5p upregulates migration and invasion of different Papillary Thyroid Carcinoma cells. BMC Cancer. 2016; 16: 108, doi: 10.1186/s12885-016-2146-z, indexed in Pubmed: 26883911.

46. Chou CK, Yang KD, Chou FF, et al. Prognostic implications of miR-146b expression and its functional role in papillary thyroid carcinoma. J Clin Endocrinol Metab. 2013; 98(2): E196-E205, doi: 10.1210/jc.2012-2666, indexed in Pubmed: 23264400.

47. Chen Xi, Ba Yi, Ma L, et al. Characterization of microRNAs in serum: a novel class of biomarkers for diagnosis of cancer and other diseases. Cell Res. 2008; 18(10): 997-1006, doi: 10.1038/cr.2008.282, indexed in Pubmed: 18766170.

48. Khordadmehr M, Jigari-Asl F, Ezzati $\mathrm{H}$, et al. A comprehensive review on miR-451: A promising cancer biomarker with therapeutic potential. J Cell Physiol. 2019; 234(12): 21716-21731, doi: 10.1002/jcp.28888, indexed in Pubmed: 31140618.

49. Goto A, Tanaka M, Yoshida M, et al. The low expression of miR-451 predicts a worse prognosis in non-small cell lung cancer cases. PLoS One. 2017; 12(7): e0181270, doi: 10.1371/journal.pone.0181270, indexed in Pubmed: 28704499.

50. Liu X, Zhang A, Xiang J, et al. miR-451 acts as a suppressor of angiogenesis in hepatocellular carcinoma by targeting the IL-6R-STAT3 pathway. Oncol Rep. 2016; 36(3): 1385-1392, doi: 10.3892/or.2016.4971, indexed in Pubmed: 27461244 .

51. Bai H, Wu S, miR-451: A Novel Biomarker and Potential Therapeutic Target for Cancer. Onco Targets Ther. 2019; 12: 11069-11082, doi: 10.2147/OTT.S230963, indexed in Pubmed: 31908476.

52. Deng Y, Zhao F, Hui L, et al. Suppressing miR-199a-3p by promoter methylation contributes to tumor aggressiveness and cisplatin resistance of ovarian cancer through promoting DDR1 expression. J Ovarian Res. 2017; 10(1): 50, doi: 10.1186/s13048-017-0333-4, indexed in Pubmed: 28743276.

53. Han Ye, Kuang Y, Xue X, et al. NLK, a novel target of miR-199a-3p, functions as a tumor suppressor in colorectal cancer. Biomed Pharmacother. 2014; 68(5): 497-505, doi: 10.1016/j.biopha.2014.05.003, indexed in Pubmed: 24972723.

54. Ghosh A, Dasgupta D, Ghosh A, et al. MiRNA199a-3p suppresses tumor growth, migration, invasion and angiogenesis in hepatocellular carcinoma by targeting VEGFA, VEGFR1, VEGFR2, HGF and MMP2 Cell Death Dis. 2017; 8(3): e2706, doi: 10.1038/cddis.2017.123, indexed in Pubmed: 28358369.

55. Mutalib NSAb, Yusof AM, Mokhtar NM, et al. MicroRNAs and Lymph Node Metastasis in Papillary Thyroid Cancers. Asian Pac J Cancer Prev. 2016; 17(1): 25-35, doi: 10.7314/apjcp.2016.17.1.25, indexed in Pubmed: 26838219 